was not improved with pulsatile perfusion, the authors state that further studies are warranted, especially given that the kidneys undergoing pulsatile perfusion in this study were from older donors with more risk factors for graft failure than those subjected to cold storage.

Original article Matsuoka L et al. (2006) Pulsatile perfusion reduces the incidence of delayed graft function in expanded criteria donor kidney transplantation. Am J Transplant 6: 1473-1478

\section{Peritoneal dialysis as effective as hemodialysis after renal graft failure}

The number of kidney transplant recipients has risen over the last few decades, leading to an increase in the number returning to dialysis after graft loss. The best treatment option for these patients is unclear; however, having experienced a period of independence following successful transplantation, such patients may prefer peritoneal dialysis (PD) at home to in-center hemodialysis (HD).

A retrospective single-center study was carried out to determine whether PD is an appropriate treatment for renal transplant recipients returning to dialysis. All patients commencing PD $(n=21)$ or HD $(n=39)$ after graft failure, up to 1 October 2002, at the University Hospital Gasthuisberg, Leuven, Belgium, were included in the analysis. Information was gathered until death, retransplantation, crossover to HD or PD, or until 1 January 2003. A higher incidence of peripheral vascular disease in the PD cohort was the only significant difference in the groups' baseline characteristics.

Overall, de Jonge et al. found no significant difference in outcomes between subjects receiving $\mathrm{PD}$ or HD; however, the HD group accrued more cardiovascular comorbidities. There was a propensity towards higher patient survival in the PD group, members of which tended to undergo retransplantation more frequently. The authors conclude that $P D$ is a safe form of renal replacement therapy following failure of a kidney graft, and is likely to be as effective as HD.

Original article de Jonge $\mathrm{H}$ et al. (2006) Comparison of peritoneal dialysis and haemodialysis after renal transplant failure. Nephrol Dial Transplant 21: 1669-1674

\section{Many patients don't reach targets despite implementation of latest bone disease guidelines}

In 2003, guidance on managing bone metabolism in chronic kidney disease was published as part of the National Kidney Foundation's Kidney Disease Outcomes Quality Initiative (NKF-KDOQI). To determine whether guideline implementation increased the likelihood of attaining recommended targets, Arenas et al. evaluated 342 patients receiving thrice-weekly dialysis at three units in Spain.

In an effort to meet the new KDOQI target for serum calcium (2.10-2.38 mmol/l [8.4-9.5 mg/dl]), there was a significant reduction in use of calciumbased phosphate binders; use of sevelamer hydrochloride-a non-calcium-based phosphate binder-rose concomitantly $(P<0.001$ for both). Increased use of dialysate with a lower calcium concentration also contributed to the greater percentage of patients whose serum calcium level fell within the new recommended range $(46.6 \%$ in 2004 vs $38.7 \%$ in 2003; $P<0.01)$.

Decreased mean serum calcium concentration correlated with increased mean serum level of parathyroid hormone (which increased from $201.4 \mathrm{ng} / \mathrm{l}$ to $311.8 \mathrm{ng} / \mathrm{l}$ following guideline implementation; $P<0.001)$. Fewer patients, therefore, met the KDOQI target for parathyroid hormone (150-300 ng/l), although this decrease was not significant. No change in average serum phosphorus level was detected.

Despite some improvements following KDOQI guideline implementation, the bone mineral metabolism parameters of many subjects remained outside recommended ranges. Arenas et al. conclude that, if the 2003 guidelines are to be implemented, therapies such as vitamin D analogs with lower hypercalcemic potential, calcimimetic agents or daily hemodialysis will be required to balance the benefits of a lower risk of hypercalcemia against the increased risk of hyperparathyroidism.

Original article Arenas MD et al. (2006) Application of NKF-K/DOQI clinical practice guidelines for bone metabolism and disease: changes of clinical practices and their effects on outcomes and quality standards in three haemodialysis units. Nephrol Dial Transplant 21: 1663-1668 